\title{
Variability and uncertainty of Track Circuit band-pass modeling for interference evaluation
}

\author{
Andrea Mariscotti \\ Università di Genova, Via Opera Pia 11A, 16145 Genova, Italy
}

\begin{abstract}
When evaluating track circuits (TCS) interference, the latest normative approach indicates the use of band-pass filters that emulate the TC receiver response. The rms value of the output, when compared to the interference limit, indicates whether a specific signal might create interference or not. The implementation of the filter has some degrees of freedom and needs thus to be characterized in terms of added uncertainty to the interference evaluation process. The latter is relevant to safety, strictly related to the incorrect assignment of the status of the track section monitored and protected by the TC itself. When giving a definitive answer about the immunity of a specific TC and the compatibility with the tested rolling stock, it is thus necessary to completely evaluate the uncertainty associated to the chosen model and to the related signal processing operations, even if - it is recognized - their influence may be considered of second order with respect to infrastructure and rolling stock non-idealities.
\end{abstract}

Keywords: Conducted Interference; Guideway Transportation Systems; Power Quality; Spectral analysis; Supply transients; Time domain analysis

Citation: Andrea Mariscotti, "Variability and uncertainty of Track Circuit band-pass modeling for interference evaluation", Acta IMEKO, vol. 2, no. 1, article 10, August 2013, identifier: IMEKO-ACTA-02(2013)-01-10

Editors: Paolo Carbone, University of Perugia, Italy; Ján Šaliga, Technical University of Košice, Slovakia; Dušan Agrež, University of Ljubljana, Slovenia

Received January $13^{\text {th }}, 2013$; In final form June $10^{\text {th }}, 2013$; Published August 2013

Copyright: (c) 2013 IMEKO. This is an open-access article distributed under the terms of the Creative Commons Attribution 3.0 License, which permits unrestricted use, distribution, and reproduction in any medium, provided the original author and source are credited

Funding: none reported

Corresponding author: Andrea Mariscotti, e-mail: andrea.mariscotti@unige.it

\section{INTRODUCTION}

When a new locomotive is introduced on existing lines it is essential to ensure that the locomotive and the signalling systems are compatible under normal operation and exceptional conditions. A key point is the evaluation of the interference mechanism, where the locomotive is the source of the disturbance (and new locomotives equipped with electronic static converters have pushed the spectrum of disturbances at higher frequency), the signalling circuit is the victim (new signalling systems operate in a wide frequency range at power frequency and audio-frequency up to some tens of $\mathrm{kHz}$ ) and the line and track are the means of propagation. Several are the relevant parameters and the degrees of freedom, related to:

- the infrastructure parameters that modify in terms of transfer function and impedance the return path of the current leaving the train axles (called "cold path") and the supply circuit feeding current to the pantograph (called "hot path") [1]-[3];

- the complex architecture of the on-board converters and drives, for traction and auxiliaries, the related static and dynamic operations, considering in particular on transient conditions [4][5];
- the susceptibility of the victim track circuits (TCs), not only for steady conditions (that can be simulated at the manufacturer's workshop), but also including various types of transients and the variability of the characterizing parameters [2][3][6].

Different vehicles or trains absorbing current in the same supply section represent a possible and relevant situation: the return current components (flowing through the analyzed track circuit section) may be led back to the respective sources with different summation rules to account for the phase relationship (depending on the degree of synchronization of the sources, the relative distance and the frequency response of the traction line) [7]. While the absolute limit on the disturbing current in the TC operating bandwidth is set by the characteristics of the TC itself with the due safety margins, the limits for each source are normally apportioned by the standardization bodies and infrastructure owners, following justified summation rules [3] and adding further safety margins.

The entire process of combined testing is particularly critical if the exigency of a high degree of reproducibility and repeatability is considered, in particular to ensure the interoperability of rolling stock and the equivalence of the test 
results by different operators and manufacturers, to address the cross-acceptance of rolling stock on existing traction lines in Europe. While the variability of infrastructure parameters and related uncertainty was already considered in the past [1], the focus of this paper is on the processing circuits and algorithms indicated by the European standards [2][3] to model the susceptibility of the victim TC and applied to the recorded pantograph current and its spectrum. A TC has an intrinsic band-pass behavior due to the internal filters and protection circuits. The standardized method for the evaluation of interference is to model the TCs by means of an equivalent band-pass filter built around the nominal TC parameters [2][3]. The processing method used for testing can also be applied in real time, while the locomotive is subject to tests or to monitor normal service.

\section{PROBLEM DESCRIPTION}

The problem considered in this paper is reduced to two elements, the victim (represented by the TC and its susceptibility) and the source (represented by the rolling stock, by its conducted emissions and the variability of their spectral properties). The interest is in quantifying the uncertainty of the test outcome with respect to the degrees of freedom offered by the implementation of the band-pass filter that models the specific TC.

\subsection{Signalling circuit fundamentals}

The considered signalling circuits are track circuits (TCs), all based on a common operating mechanism, that of transmitting a modulated signal from a transmitter (TX) to a receiver (RX) over the track, and sensing track occupation by measuring the amount of signal that reaches the RX. The track is detected as occupied, when the rails are shunted by the low resistance axles of the entering rolling stock, if the RX signal drops below a given threshold. The TCs frequency interval ranges from some tens of $\mathrm{Hz}$ for power frequency TCs up to about $20 \mathrm{kHz}$ for audio-frequency TCs, with only a few exceptions. The longitudinal voltage drop along the track is mainly inductive and is thus roughly proportional to the frequency; for this reason, to maximize the amount of signal reaching the RX in free conditions, some techniques are normally adopted (capacitive compensation of the track impedance, matching of TX impedance to track impedance, directionality of TX signal towards the respective RX by use of track bonds) [8]. The signal is nowadays always modulated to increase the robustness against the superimposed conducted disturbance of circulating rolling stock, that may occur in the operating band of the TC itself. Moreover, the RX has an intrinsic band-pass behavior determined, first, by the electrical coupling method itself often obtained by using resonant circuits tuned including the track itself, and, second, by additional analog or digital filters to increase noise rejection. The so-called relays connected to the $\mathrm{RX}$ output cable in the interlocking cabin are responsible for additional processing, since they often include a delay, to filter out transient components (such as in motor relays or slow pickup relays) or a phase/frequency matching circuit between the track and a "clean" parallel cable between TX and RX (such as for vane relays). The modern relays are digital implementations on an interlocking computer and their functions are even more extended; however, the continuity with respect to "traditional functions", often required by railways, has led to standardization on old functionalities.
An entire TC is a complex, potentially non-linear, system that can be characterized with a black box approach by a series of curves of susceptibility, only if a certain degree of approximation is acceptable. Susceptibility tests with steady signals are easier, in that swept sinusoidal signals are applied first, and then followed by multi-sine signals, all of variable amplitude, until the effects of interference are detected. If transient signals are considered too, the characterization is more complex, since the time-frequency properties of the signal and the TC time domain response are strongly related, and the identification of the suitable subspaces, indexes and mathematical representations is still under investigation [3]. Pragmatically, in this paper, the band-pass approach followed by the cited standards is considered, thus fixing the method of evaluation, while the uncertainty related to the variability of the applied steady and transient signals is analyzed.

\subsection{Rolling stock}

The rolling stock (i.e. a locomotive or electrical multiple units) represents the source of disturbance as the return current leaving its axles and coupling along the track with the victim TC. Coupling is conductive and occurs mainly in differential mode, while the return current leaves in principle symmetrically the axles, so representing a common mode variable. A certain amount of asymmetry exists in the rail-wheel contact resistances, in the rail self and mutual impedance and in the grounding electrical terms, and represents a first important assumption for an administration or standardization committee, while fixing the safety margins for the evaluation methods and limits.

Moreover, the pattern of conducted emissions is basically determined by the characteristics of the on-board converters and of the adopted modulation schemes. Even in perfectly steady conditions, modulations can change in an attempt of optimization, depending on the motor speed and torque, or even pantograph voltage level. Normal rolling stock operations are divided into acceleration, coasting and braking; acceleration and braking may occur at different rates, and for braking a regenerative action is possible up to a maximum allowed line voltage, then dissipative electric braking is applied; below a certain speed, on the contrary, braking is normally pneumatic. Coasting at a fairly constant speed may occur at different line slopes, positive and negative, so with different amounts of however small absorbed power.

Transient conditions may be various, such as pantograph bounce, wheel slip and slide and input transformer and filter inrush current. The former causes a series of electric transients in the input circuitry, in particular transformer and filters, influencing also traction and auxiliary converters. The second may be quite complex, in particular if an anti-skidding mechanism is implemented, quickly changing the traction converter modulation. The latter occurs when the rolling stock is stationary and raises its pantograph (or more properly closes its main circuit breaker), or while passing neutral sections (similar to pantograph bounce). In the presence of transients (thus non-stationary signals) a Fourier based analysis may be inadequate and unsatisfactory, taking into account in particular the trade-off between the time duration of the transient, the desired frequency resolution and the time constants of the RX and relays. 


\subsection{Traction supply systems and track circuits}

Rolling stock may circulate on different supply systems: dc systems at 1500 and $3000 \mathrm{~V}$ rated voltage and ac systems at $16.7 \mathrm{~Hz} / 15 \mathrm{kV}$ (used in German speaking countries and also in Sweden and Norway) or at $50 \mathrm{~Hz} / 25 \mathrm{kV}$ (for many railway systems, of the high speed type or conventional). While in the past a given rolling stock was bounded to one or few countries with the same supply system, several modern locomotives and EMUs can be supplied by two (or even three, recently) different supply systems. This has raised the problem of interoperability even more and of the compatibility of variable combinations of supply system frequency, type of locomotive and type of track circuit.

While there are evident impediments for the use of a $50 \mathrm{~Hz}$ TC under a $50 \mathrm{~Hz}$ supply system, track circuits and supply systems combinations are all possible, even if for historical reasons and opportunities only a subset of them is really implemented [6]. However, some TCs equip for example only high speed lines or some others are not put by the administration in the list of the preferred ones [3][6]. In any case the exhaustive verification of compatibility of a new locomotive or EMU against the whole set of the installed TCs is an expensive and time consuming mission; moreover, any rolling stock unit requires an initial period of preliminary verifications and fine tuning that can be performed in-house. This justifies the approach followed by CENELEC of a shared framework for TC modeling, where the TC band-pass behavior is simulated by adopting the correct band-pass filter implementation.

\section{SETUP FOR TRACK CIRCUIT INTERFERENCE TESTING}

Test methods generally assess receiver immunity through signal processing of the recorded variables. A distinction may be made between type tests performed on reference railway tracks with the mentioned test methods and on-line real time monitoring of traction return current to prevent signalling interference. On-line monitors implement the same limit curves and processing methods, and even the same algorithm, used for type testing and they are aimed at detecting possibly critical interference situations in real time. When a locomotive is undergoing the certification process to run inside some country, circulation is restricted and authorized during the many tests if such a monitor is installed on-board, to prevent hazardous situations due to excessive disturbance onto the infrastructure. In general, an administration might require that any locomotive or unit running on the network is permanently equipped with monitoring equipment for an increased level of safety.

The considered track circuit receivers have all a band-pass behavior. The band-pass function may be obtained with different solutions: an analogue filter, a digital filter or a Discrete Fourier Transform (DFT). In general the analogue solution is not preferable for reasons of flexibility and implementation costs. The CLC/TS 50238-2 [3] proposes the digital band-pass filter solution and this will be considered here, against a set of synthetic and real test signals. In the 50238 standards also the use of DFT is considered, as dictated by some national standards, but not recalled explicitly in the last issue [3].

The standardized filter for the evaluation of interference has some degrees of freedom in its specification, also depending on the specific target $\mathrm{TC}$ :
- attenuation or frequency response and filter order are the most relevant and obvious; attenuation is normally given in one or two frequency points on the sides of the filter band, thus defining its bandwidth (at $-3 \mathrm{~dB}$ points) and its roll-off (e.g. adding another point at -6 or $-10 \mathrm{~dB})$;

- the filter type is sometimes specified, thus fully identifying the filter behavior, and including the phase response, that is particularly relevant for transients and for the correct combination of the sinusoidal components located within the filter band.

The block diagram of the interference evaluation system (IES) includes an input band-pass filter (BPF) followed by a routine for the computation of the total $\mathrm{rms}$ in the filter band (RMS detector), and is shown in Figure 1. The signals are indicated here as continuous time signals, even if they are in reality discrete time signals.

RMS computation of the BPF output $y$ may be in general performed in two ways: in the frequency domain the root of the sum of the squared DFT components is used; in the time domain the exact definition of $\mathrm{rms}$ is used, taking the average of $y^{2}$. The latter is preferred here because it is closer to the rms definition and the integration time $T_{i}$ specified in [3] can be readily implemented (using modified Simpson rule over a moving integration window of amplitude $T_{i}$ ).

The test signal that will be used in the following is the absorbed pantograph current $i(t)$. Real signals are the result of recordings on various locomotives and trains and cover real situations, including any type of transients as they occur in normal circulation conditions or purposely generated. Synthetic signals are conceived to be similar to known already observed spectral signatures, but with controllable parameters, to test the sensitivity of the filter response to their variations.

The BPF, when a precise specification is lacking, may be implemented in any of Infinite Impulse Response (IIR) architectures, provided that the requisites on attenuation, rolloff and number of poles are met [6][9]. In some cases the TC band-pass behaviour is specified by the administration or the manufacturer that clearly indicates the required filter architecture, that is the Butterworth one; in other cases the indication is not explicit or unique, or even the specified TC frequency response cannot be led back to a "classical" IIR architecture.

\section{CHARACTERIZATION OF THE SELECTED TRACK CIRCUITS}

The set of selected victim TCs is defined in Table 1; the criteria followed for the selection consist of identifying various frequency intervals where train emissions behave differently and where different specification schemes apply [3][6]. The $\mathrm{BPF}$ transfer functions are analyzed for three different IIR

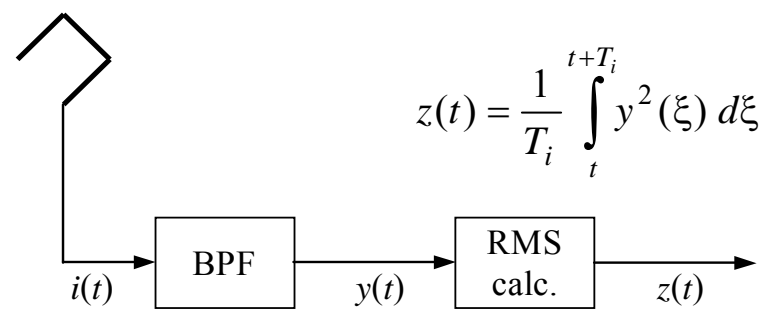

Figure 1. Block diagram of the IES with the BPF and the RMS calculation block (the possible implementations in the time or frequency domain are outlined in the upper right position). 
Table 1. Reference TCs at power and audio frequency used in the foregoing analysis.

\begin{tabular}{|c|c|c|c|c|c|}
\hline $\mathrm{TC}$ & $f_{0} / \mathrm{Hz}$ & $\Delta f / \mathrm{Hz}$ & $T_{i} / \mathrm{s}$ & $\operatorname{order} N$ & Limit/A \\
\hline \multirow{3}{*}{ 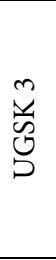 } & 208.75 & $\begin{array}{l}6.5 @-3 \mathrm{~dB} \\
14 @-20 \mathrm{~dB}\end{array}$ & 0.5 & $2 \times 3$ & 4 \\
\hline & 222.45 & $\begin{array}{l}6.5 @-3 \mathrm{~dB} \\
14 @-20 \mathrm{~dB}\end{array}$ & 0.5 & $2 \times 3$ & 4 \\
\hline & 242.15 & $\begin{array}{l}6.5 @-3 \mathrm{~dB} \\
14 @-20 \mathrm{~dB}\end{array}$ & 0.5 & $2 \times 3$ & 4 \\
\hline \multirow{8}{*}{$\vec{\vartheta}$} & 1549 & \multirow{8}{*}{ 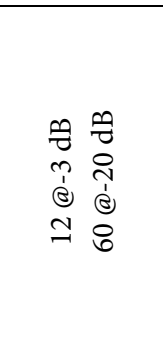 } & 2.0 & \multirow{8}{*}{ 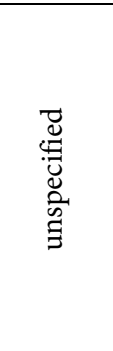 } & 0.09 \\
\hline & 1699 & & 2.0 & & 0.09 \\
\hline & 1848 & & 2.0 & & 0.09 \\
\hline & 1996 & & 2.0 & & 0.09 \\
\hline & 2146 & & 2.0 & & 0.09 \\
\hline & 2296 & & 2.0 & & 0.09 \\
\hline & 2445 & & 2.0 & & 0.09 \\
\hline & 2593 & & 2.0 & & 0.09 \\
\hline
\end{tabular}

architectures, Bessel (BS), Butterworth (BW) and Chebyshev (CB): the filter order is the same and the bandwidth is adjusted for i) equivalence (see below the two criteria used, the first with respect to the $-3 \mathrm{~dB}$ points only, the second including the rest of the bandwidth by means of the Equivalent Noise Bandwidth, ENBW, estimation) and ii) to comply with the attenuation specifications of Table 1.

The two TCs selected for the analysis feature either a direct BW implementation (UGSK 3) or an unsatisfactory implementation within the IIR framework (TI 21).

A synthetic noise signal with a flat power spectral density in the filter band is first used to derive the basic BPF properties and to preliminarily rate the performance of the different BPF implementations.

\subsection{TC with implicit Butterworth implementation}

In the first example (focusing on UGSK 3), the three BPF architectures have all the same number of poles $(n=3)$ and the same central frequency $\left(f_{c}=208.75 \mathrm{~Hz}\right)$. The frequency response is shown in Figure 2. Concerning the definition of filter bandwidth and the equivalence between the three

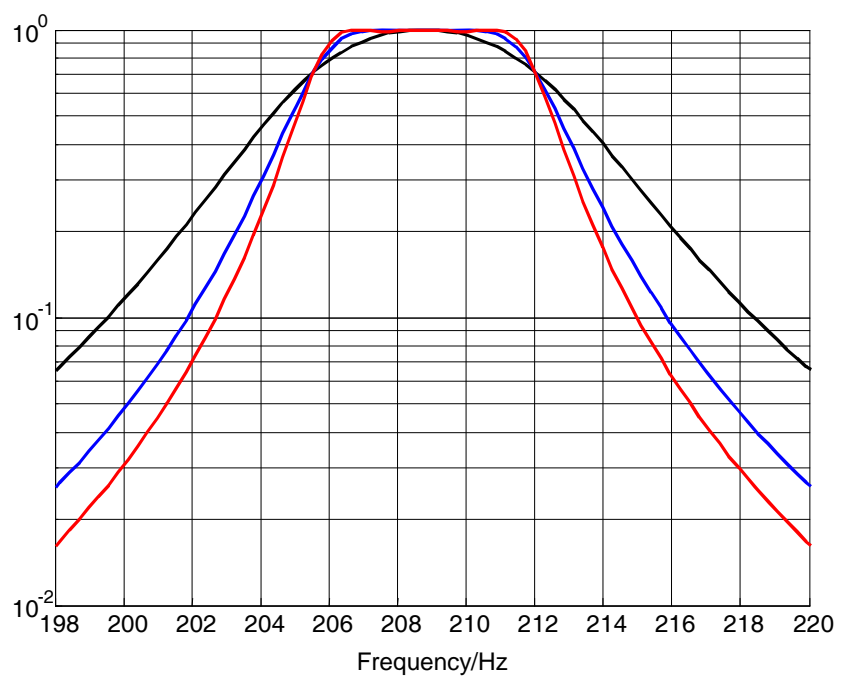

Figure 2. Frequency response of the three BPF implementations for UGSK 3: BS (black), BW (blue), CB (red).
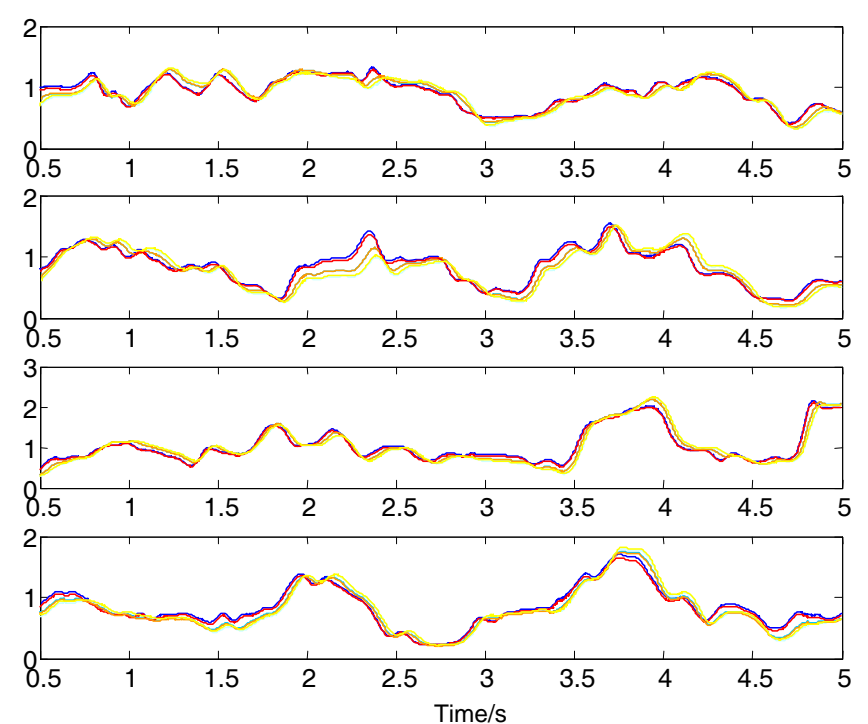

Figure 3. Calculated rms signal $z(t)$ for UGSK 3 and four random sequences: "-3 dB criterion" (blue) and "ENBW criterion" (red); BS (dark), BW (medium), CB (light).

architectures, two criteria were followed (the equivalence is established either for equal $-3 \mathrm{~dB}$ attenuation or for equal Equivalent Noise Bandwidth) and two series of tests were performed. The ENBW difference for the $-3 \mathrm{~dB}$ criterion is $2.83 \%$ between BS and BW and $1.14 \%$ between $\mathrm{CB}$ and $\mathrm{BW}$, the BW always taken as reference. The condition of $-3 \mathrm{~dB}$ bandwidth is imposed when the band-pass filter is created from the low-pass prototype: it is evident that the BW architecture meets also the specification at $-20 \mathrm{~dB}$, thus indicating the BW as the underlying filter architecture for the UGSK 3 specification.

The difference in $q(t)$ for the $-3 \mathrm{~dB}$ and ENBW bandwidth criteria was evaluated as the rms deviation divided by the average value of $z(t)$ for a set of random test sequences (see Figure 3). While there is a general agreement between the curves (accounting for not so much different rms values over the $5 \mathrm{~s}$ records), there are some small parts of the traces showing an amplitude discrepancy (like the second one between 2 and $2.5 \mathrm{~s}$, with a spread of up to $20 \%$ for the three architectures and a negligible difference between the two bandwidth criteria).

In Table 2 the results for twenty test sequences are shown in aggregate form: maximum deviation $\delta z_{\max 1}$ and $\delta z_{\text {max } 2}$ and $\mathrm{rms}$ deviation $\delta z_{z_{m s}}$ and $\delta z_{z_{m} 2}$ between BS/BW and CB/BW.

Being the difference among the three BPF in terms of ENBW less than $3 \%(2.83 \%$ and $1.14 \%$, as said above), the larger rms variability is explained with differences in the phase response.

\subsection{TC without a direct IIR implementation}

The test is repeated for the TI 21 track circuit, whose frequency response is peculiar and cannot be led back to one of

Table 2. Statistical analysis of deviations of signal $z(t)$ for the BPF architectures: rms value and maximum value over $5 \mathrm{~s}$ records for Bessel and Chebyshev, divided by the average $z(t)$ of the Butterworth implementation.

\begin{tabular}{|c|c|c|}
\hline Parameter & Bessel & Chebyshev \\
\hline$\delta z_{r m s} / z_{a v(\text { Butt. })}$ & 0.1075 & 0.0538 \\
\hline$\delta z_{\max } / z_{a v(\text { Butt. }}$ & 0.5144 & 0.2810 \\
\hline
\end{tabular}




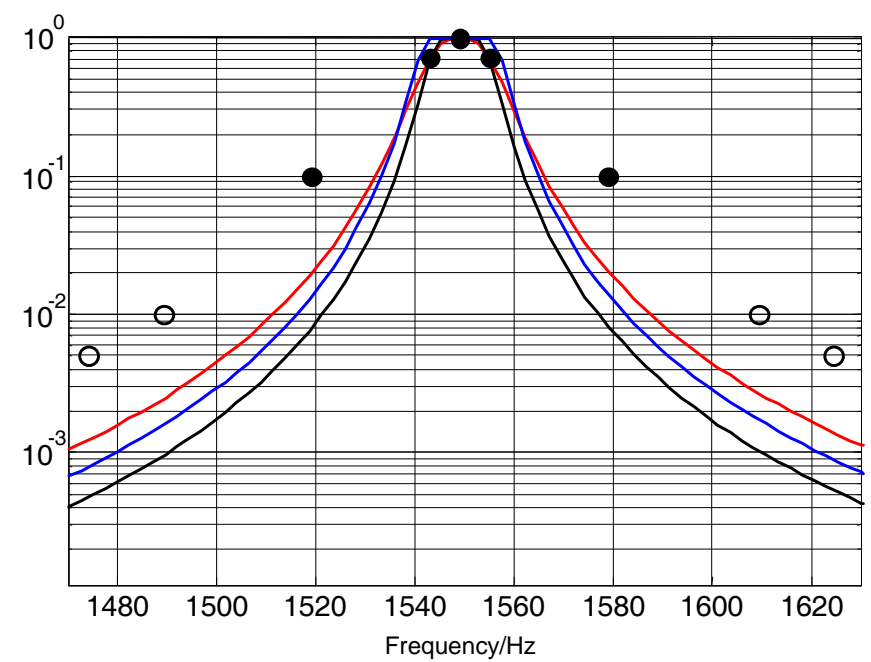

Figure 4. Frequency response of the three BPF implementations for TI 21: BS (black), BW (blue), CB (red); frequency response specifications (solid circles) and additional points (hollow circles).

the three considered IIR architectures (as shown in Figure 4). The specified TC bandwidth is remarkably larger and adopting one of the three IIR architectures would imply an underestimation of the real interference coupled onto the TC.

In this case there is no implicit or explicit indication for the $\mathrm{BPF}$ architecture and after having tried the three BS, BW and $\mathrm{CB}$ architectures, the fulfilment of the frequency response specifications is reached with a high order FIR filter, synthesized with the inverse DFT technique [9] (see Figure 5). Additional points were added to the specification of the nominal frequency response only to obtain a better shape of the roll-off slope. Different filter lengths (i.e. number of filter taps) were considered and the approximation is satisfactory for our tests beginning with $\mathrm{N}=4096$, so that any deviation from the nominal frequency response is smaller than $1 \%$ and can be considered negligible. The added frequency response points (shown as hollow circles in Figure 5) impose a stop-band amplitude of the filter frequency response below $1 \%$ of the center band amplitude (normalized to 1). The relative error between the filter and the nominal frequency responses is kept smaller than $1 \%$.

\section{TEST WITH REAL LOCOMOTIVE CURRENTS}

Some pantograph current recordings are considered: they

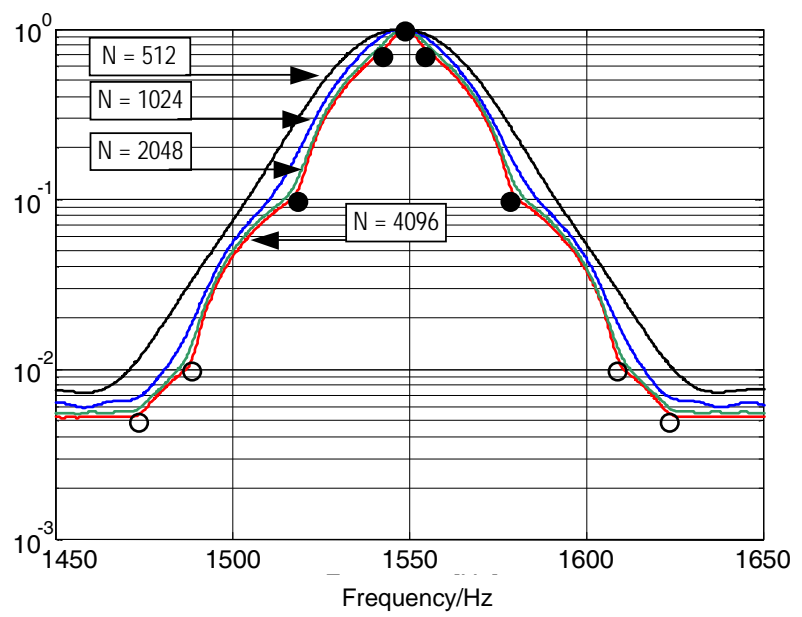

Figure 5. Frequency response of the FIR implementation for TI 21. were recorded by different types of locomotives in real operating conditions and under different supply networks. The variability due to the filter architecture and the performance of the various implementations are thus tested and evaluated in real conditions, by using both steady and transient signals. The instrumental uncertainty behind the measurement setups is irrelevant to the analysis presented in this work, related only to the uncertainty given by the chosen filter architecture and parameters. A complete analysis of the uncertainty of the measurement equipment and setup behind the used recordings can be found in [10].

\subsection{Steady pantograph current}

Some test signals are used, selected from the recordings performed on-board a German and a French train running on the respective supply networks with fundamental frequency of 16.7 and $50 \mathrm{~Hz}$ [10][11]. The signal waveforms do not show any remarkable local non-stationarity or transient behaviour, while some step changes are visible for some spectral components (see Figure 6). Spectra are calculated versus time with Short Time Fourier Transform: the frequency resolution is one third of the fundamental and a Hamming window was used to control the frequency leakage.

The spectra are characterized in general by odd harmonics of the fundamental supply frequency [10]. The low frequency portion of the spectrum of Figure 6(a) shows clearly the 11th and the 15th harmonics with amplitude of about $0.3 \mathrm{~A}$; their amplitude is quite constant over time, while the 13th harmonic is somewhat lower and has an intermittent behaviour. Similar considerations can be made for the high frequency portion located around two of the TI 21 channels.

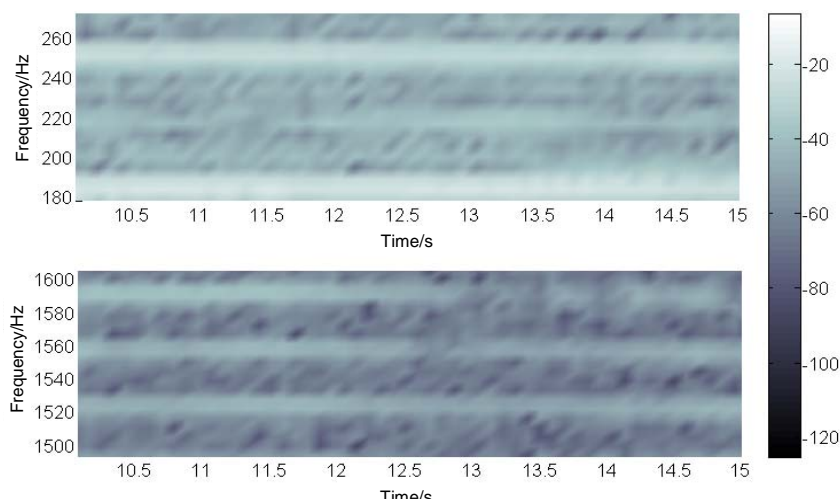

(a)

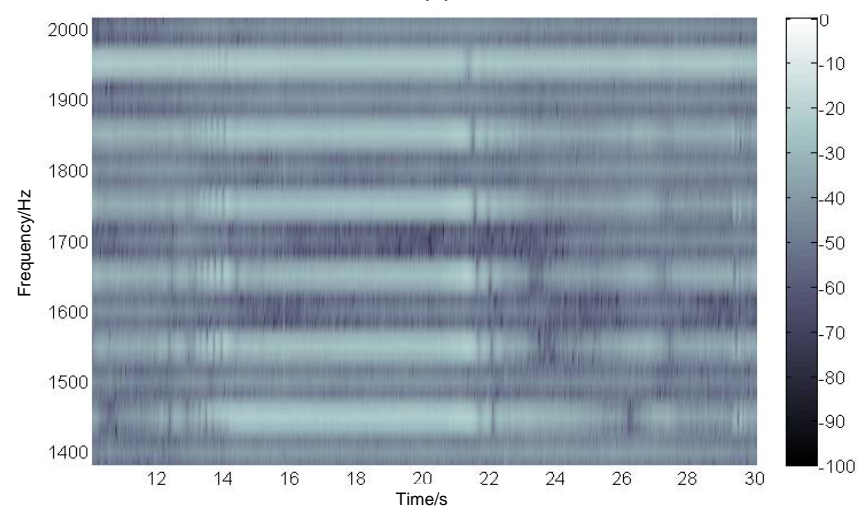

(b)

Figure 6. Short Time Fourier Transform spectra of the (a) German and (b) French pantograph current in the frequency intervals used to test UGSK 3 and $\mathrm{TI} 21$ (the intensity is expressed in dBA peak). 
Considering Figure 6(b), all the odd harmonics of the $50 \mathrm{~Hz}$ fundamental are visible and are of approximately equal amplitude. The amplitude is particularly high (nearly $1 \mathrm{~A}$ ) and the traces are shortly interrupted by rapid transients, typical of the French recordings, as described in [11]. The influence of the same interruptions may be recognized in the time domain filter response shown in Figure 7, even if attenuated by the filter transient response.

In Figure 7 the time domain response of the different implementations is shown for the two test signals: the response of the UGSK 3 filters working at the lower frequency is always characterized by the larger uncertainty for the chosen filter implementation.

The first three traces of Figure 7(a) show variability between the red, the blue and the black waveforms of up to $100 \%$. This represents a large variability that is to be accounted for in this kind of analysis. The different behaviour of the time response for the three UGSK 3 channels is explained by observing the characteristic harmonics and their sidelobes of the upper spectrum in Figure 6(a):

- the large clear horizontal band at $183 \mathrm{~Hz}$ is the $11^{\text {th }}$
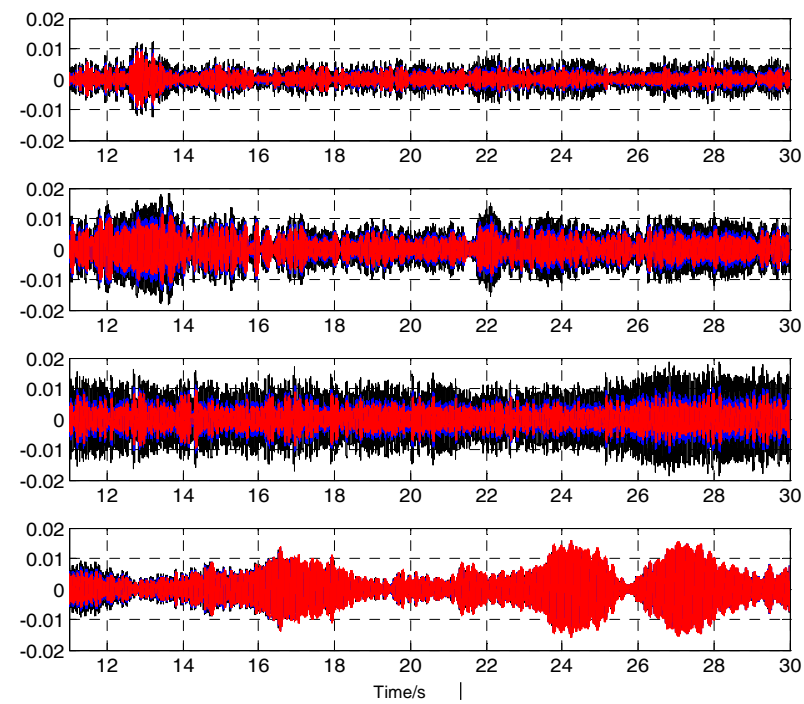

(a)
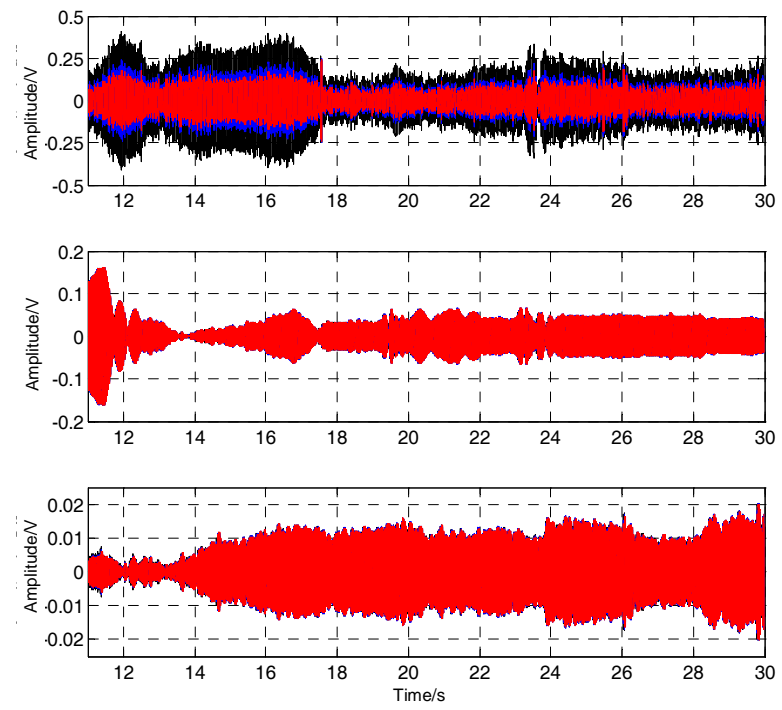

(b)

Figure 7. Time response of the three BPF implementations for (a) Germany (UGSK 3 with the $208.75 \mathrm{~Hz}, 222.45 \mathrm{~Hz}$ and $242.15 \mathrm{~Hz}$ channels, TI 21 with the $1549 \mathrm{~Hz}$ channel) and (b) France (TI 21 with the $1549 \mathrm{~Hz}, 1699 \mathrm{~Hz}$ and $1848 \mathrm{~Hz}$ channels). harmonic of the $16.7 \mathrm{~Hz}$ supply frequency, and some leakage to the $208 \mathrm{~Hz}$ channel is observed only at the end of the trace;

- the $222 \mathrm{~Hz}$ channel on the contrary is nearly centered on the $13^{\text {th }}$ harmonic, from which a larger amplitude in the filter output, characterized also by wider changes;

- the $242 \mathrm{~Hz}$ channel is in similar conditions, but at a more intense characteristic harmonic, the $15^{\text {th }}$, with a more regular amplitude profile versus time, and this explains the larger and more regular values in the output.

The spread between the three filter implementations that is observed in particular in the third channel may be explained by observing that not only, as a general fact, the phase relationship between the spectral components is important, but also that any change of the slope of the amplitude and phase response (rolloff) around the corner frequency $(242.15+3.25 \mathrm{~Hz}$ at $-3 \mathrm{~dB})$, changes the amount of the captured $15^{\text {th }}$ located at $250 \mathrm{~Hz}$. The same may be observed in the first TI 21 channel at $1549 \mathrm{~Hz}$, so centered exactly on a particularly intense characteristic harmonic of the $50 \mathrm{~Hz}$ French system, from which the largest amplitude of the filter output and the spread due to different roll-off at the corner frequency. The same channel under a 16.7 $\mathrm{Hz}$ supply (last trace of Figure 7(a)) features a ten times smaller interference and less variability for the chosen filter implementation, due to the smaller content of high frequency harmonics of the $16.7 \mathrm{~Hz}$ supply systems.

\subsection{Transient pantograph current}

The analysis of sec. 5.1 is repeated considering transients observed in the pantograph current during tests performed in Sweden on a $16.7 \mathrm{~Hz}$ supply system in 2008. Scheduled ramps up and down were applied, so non-stationarity is expected, at least localized on some spectrum components. During the tests some short-time transients occurred as well, such as pantograph bounces, due to the mechanical interaction of the catenary and pantograph systems. The pantograph current waveforms used are shown in Figure 8 and their spectra in Figure 9; rapid changes of the amplitude of some harmonic components are visible in particular in Figure 9(a). Tests are performed on the
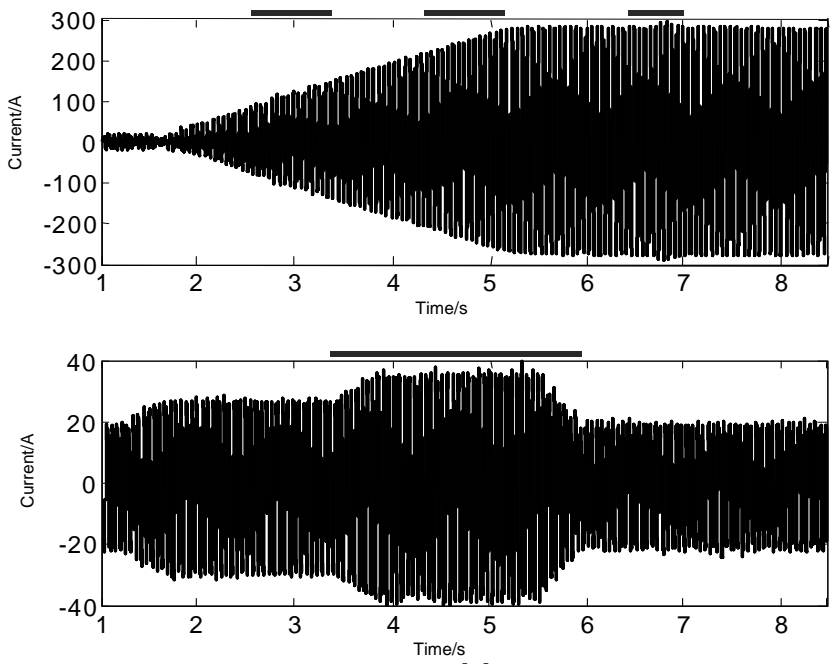

Figure 8. Pantograph current waveforms used for testing the UGSK 3 filter implementations; time intervals where transients can be observed are marked by a horizontal grey line; the two graphs show different kind of transients while accelerating from standstill (top) and during sudden changes of the driving/catenary conditions. 


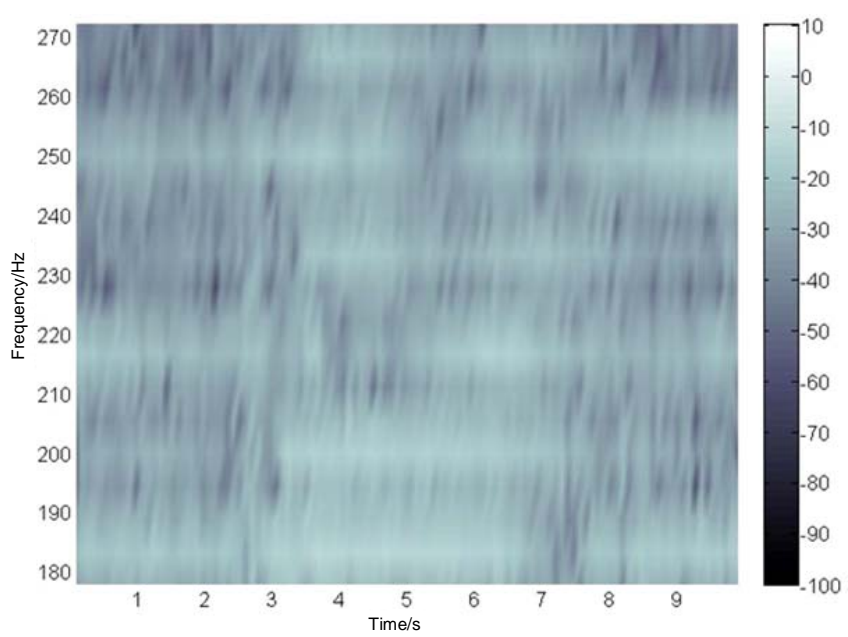

(a)

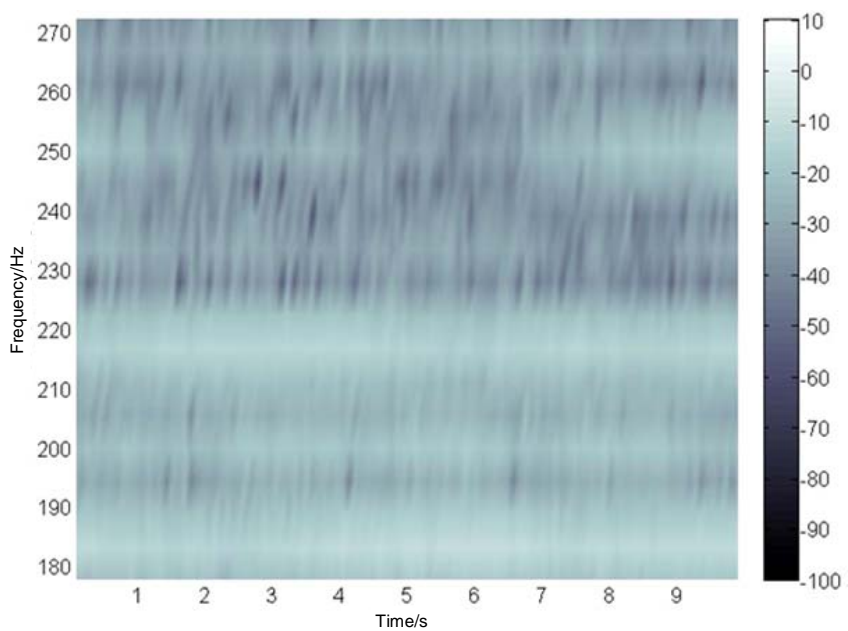

(b)

Figure 9. Short Time Fourier Transform spectrum of the Swedish pantograph current in the frequency intervals used to test UGSK 3 (the intensity is expressed in dBA peak).

UGSK 3 channels at $208.75 \mathrm{~Hz}, 222.45 \mathrm{~Hz}$ and $242.15 \mathrm{~Hz}$.

In Figure 10 the rms output is shown, obtained by time window integration of the squared output, as indicated in Figure 1. The curves of Figure 10(a) show the step changes due to the heavy transient behaviour of some spectral components, in particular around 3,4 and 6.5 seconds. The integration time $T_{i}$ is $2 \mathrm{~s}$ for the UGSK 3 track circuit receiver and this introduces a time delay in the rms output response, that may be slightly variable depending on the time behaviour of the specific spectral components on the specific channel: for this reason the peaks of the rms output $z$ of the three channels might be not exactly aligned in time.

A large spread is observed, up to $100 \%$ for BS, $30 \%$ for CB, both during short and long time intervals, as it can be seen in Figure 10(a) around $6.5 \mathrm{~s}$ and in Figure 10(b) over the entire time interval for the first two channels.

During transients, extraneous components may appear and the existing ones are subject to frequency leakage, from which the larger spread of the Bessel architecture that features the highest sidelobes. On the contrary, what is observed in the output cannot be put in direct relationship with the ENBW that is not so different for the three IIR filter architectures. The supply harmonics occupy a large percentage of the frequency interval and there is no much space left between them, so that
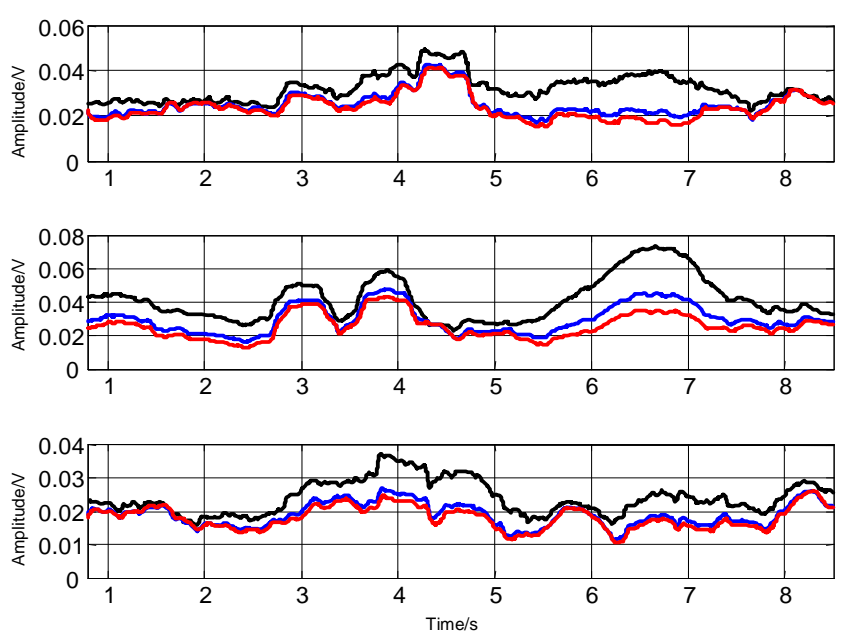

(a)
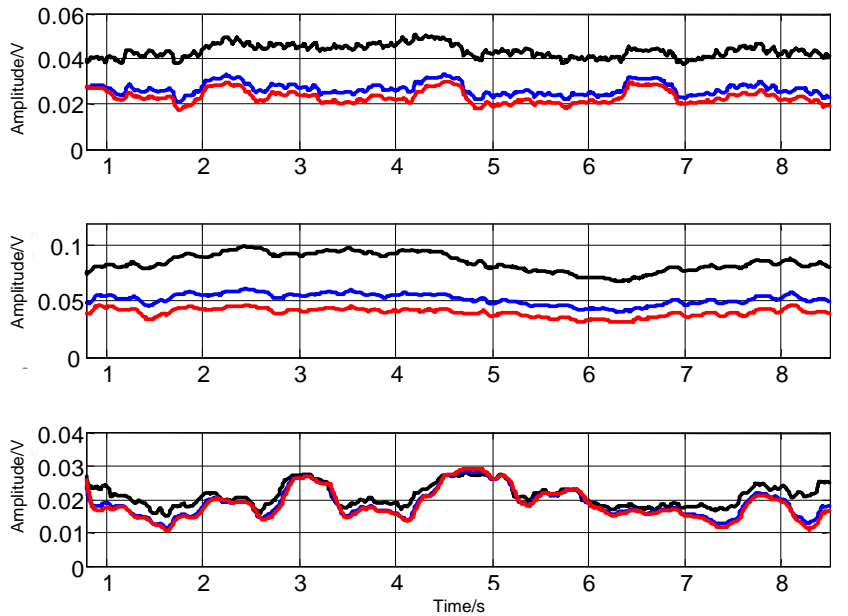

(b)

Figure 10. Time response of the three BPF implementations for the Swedish transients for UGSK 3 with the $208.75 \mathrm{~Hz}, 222.45 \mathrm{~Hz}$ and $242.15 \mathrm{~Hz}$ channels.

steep sidelobes more than any other feature are relevant for a TC manufacturer.

\section{CONCLUSIONS}

The focus of this work is the evaluation of the uncertainty related to the choice of the implementation of the band-pass filter that simulates track circuit behaviour. The rms value of the band-pass filter output is used for the evaluation of conducted disturbance produced by rolling stock in the track circuit operating bands, as dictated by standards [2][3]. The tests are carried out using synthetic noise sequences and real pantograph current waveforms.

This uncertainty, related to the post-processing of the recorded signal in order to assess possible interference to the safety relevant track circuit systems, is one of the terms of the uncertainty budget and in some cases is not negligible if compared to other elements of the measurement chain.

The variability of the rms value $z(t)$ of the filter output evaluated with respect to the Butterworth architecture using synthetic test signals gave $10.8 \%$ and $5.3 \%$ for Bessel and Chebyshev implementations, respectively.

The application of real waveforms of recorded pantograph currents have shown a variability in the output response of the three BPF implementations of up to $100 \%$. The Bessel implementation shows the largest response due to its highest 
sidelobes, which do not allow filtering out the leakage of supply harmonics and other non-harmonically related components during transients. It is thus sensible to assume that a track circuit manufacturer implements a more selective frequency response for its product, that is best simulated by a Butterworth or Chebyshev architecture. The TI 21 track circuit is an exception. The frequency response curves of Butterworth and Chebyshev may still exhibit in some cases a deviation of up to $30 \%$, but only a few $\%$ on average.

It is thus evident that specifying the attenuation at a few frequency points is an insufficient characterization of the track circuit, for which the required band-pass filter architecture should always be specified by the standards.

\section{REFERENCES}

[1] A. Mariscotti, "Distribution of the traction return current in AC and DC electric railway systems", IEEE Transactions on Power Delivery, vol. 18, Oct. 2003, pp. 1422-1432.

[2] "CENELEC Std. FprEN 50238-2, Railway applications Compatibility between rolling stock and train detection systems - Part 2: Compatibility with track circuits", 2008-06.

[3] "CENELEC Std. CLC/TS 50238-2, Railway applications Compatibility between rolling stock and train detection systems - Part 2: Compatibility with track circuits", 2010-07.

[4] A. Steimel, "Electric Traction - Motive Power and Energy Supply: Basic and Practical Experience", Oldenbourg Industrieverlag, Munich, Germany, 2008.
[5] CCITT (International Telegraph and Telephone Consultative Committee), 1989, "Directives concerning the protection of telecommunication lines against harmful effects from electric power and electrified railway lines", Volume IV (ISBN 92-6104051-9), Geneva, Switzerland.

[6] "CENELEC Std. CLC/TR 50507, Railway applications Interference limits of existing track circuits used on European railways", 2007-05.

[7] B. Hemmer, A. Mariscotti, D. Wuergler, "Recommendations for the calculation of the total disturbing return current from electric traction vehicles", IEEE Transactions on Power Delivery, vol. 19, April 2004, pp. 1190-1197.

[8] A. Mariscotti, M. Ruscelli and M. Vanti, "Modeling of Audiofrequency Track Circuits for validation, tuning and conducted interference prediction", IEEE Transactions on Intelligent Transportation Systems, vol. 11, March 2010, pp. 52-60.

[9] S.K. Mitra, "Digital Signal Processing - A Computer Based Approach", 1 1st ed., McGraw-Hill, New York, 1998.

[10] A. Mariscotti, "Direct Measurement of Power Quality over Railway Networks with Results of a $16.7 \mathrm{~Hz}$ Network", IEEE Transactions on Instrumentation and Measurement, vol. 60 n. 5, May 2011, pp. 1604-1612.

[11] A. Mariscotti, "Results on the Power Quality of French and Italian 2x25 kV $50 \mathrm{~Hz}$ railways", I2MTC 2012, Graz, Austria, May 13-16, 2012. 